\title{
ALGUNAS OBSERVACIONES SOBRE LA MORTALIDAD POR CAUSAS EN MÉXICO, 1950-1967
}

\author{
Francisco Alba H. y Ricardo Alvarado \\ El Colegio de México
}

\section{INTRODUCCIÓN}

El estudio de las causas de muerte interesa diversamente a una serie de ciencias: medicina, salud pública, demografía. El punto de vista de la demografía es el mismo que tiene frente al más general de la mortalidad. Interesa a la demografía "la forma en que las características físicas o biológicas, la organización social y el medio ambiente se relacionan con la mortalidad". 1

Tanto los factores biológicos como los sociales -en su sentido más amplio, que incluiría la acción de éstos sobre los factores ambientales- son determinantes de un nivel dado de mortalidad, de una estructura dada de causas de defunción. Los primeros son básicos, en cuanto que la sobrevivencia siempre estará enmarcada dentro de los límites de la "mortalidad biológica". Dentro de estos límites es posible la acción de los factores sociales. Sin intención de hablar de límites precisos, la mortalidad tendría dos componentes: uno endógeno - causas de muerte intrínsecas al organismo- y otro exógeno - causas de muerte cuyo origen son agentes externos al organismo.

El estudio de los factores asociados a la mortalidad evidencia ciertas relaciones estadísticas entre estructuras de la mortalidad por causas y los niveles de desarrollo social, cultural y económico. Estas relaciones se producen por el hecho de que ciertas causas de muerte -las exógenas- pueden considerarse "controlables" por el avance en el desarrollo social.

En consecuencia, el estudio de las causas de muerte resulta ser un instrumento más de investigación en el análisis de la mortalidad misma y de los niveles de desarrollo de la sociedad. La historia de la sociedad humana, hasta los inicios de la época moderna, estuvo caracterizada por una elevada mortalidad causada por enfermedades provocadas principalmente por agentes externos al organismo humano o por carencias: epidemias y plagas, guerras, hambres. En la era moderna, con sus revoluciones agricola e industrial, social y cultural, algunas sociedades mejoran su dotación de alimentos, su alojamiento, su higiene personal y pública, sus condiciones de vida en general; ello

1 Juan C. Elizaga, Métodos demográficos para el estudio de la mortalidad, CELADE, Santiago de Chile, 1969, p. 9. 
permite un primer control de enfermedades endémicas y transmisibles que se refleja en un lento declinar de la mortalidad.

Después vendrían el desarrollo de la medicina y de la salud pública, los progresos de las técnicas quirúrgicas y de diagnóstico, las formas institucionales de asistencia y previsión. El control sobre la muerte se extendió a un mayor número de causas, hasta alcanzar incluso enfermedades de carácter endógeno. ${ }^{2}$

El proceso ha requerido en su realización varios siglos y sólo incluye el mundo "desarrollado". En el mundo "subdesarrollado" el proceso ha sido diferente; su inicio es reciente, su ritmo rápido y los factores asociados diferentes. La disponibilidad e importación de métodos baratos y efectivos en el control masivo de la mortalidad ha sustituido parcialmente las mejoras en las condiciones de vida en este proceso de resistencia y control de las enfermedades "evitables".

Tal vez sea posible en la actualidad alcanzar niveles regulares o relativamente bajos en la mortalidad con poco mejoramiento en las condiciones socioeconómicas de la población. Evidentemente existe un nivel difícil de sobrepasar sin cambios fundamentales en esas condiciones. Incluso en los países "desarrollados" los máximos niveles (alcanzables) de salud dependen de qué tan efectivamente estén distribuidos los beneficios de la sociedad urbano-industrial entre su población.

Si se acepta, pues, que la estructura de la mortalidad por causas depende de la implementación de medidas de salud pública, de los niveles de desarrollo y de los progresos médico-asistenciales, su análisis es útil como indicador del estado de desarrollo y de la salud pública alcanzados por la sociedad. ${ }^{3}$

Este análisis es de utilidad para el estudio de la mortalidad misma, máxime en las actuales circunstancias en que los indicadores brutos del nivel de la mortalidad encubren grandes disparidades debido, básicamente, a la influencia que sobre tales indicadores provocan las estructuras diferentes de edad de las poblaciones. Existe además, como ya se indicó, una clara relación entre niveles de mortalidad y estructuras de causas de muerte: "Cuando la mortalidad desciende - dice Elizaga - pierden importancia relativa las muertes por enfermedades infecciosas y parasitarias, por gastroenteritis y por enfermedades agudas del aparato respiratorio (gripe, neumonía), por ejemplo; y aumenta, en cambio, la importancia relativa de las muertes debidas a cáncer y a enfermedades cardiovasculares". ${ }^{4}$ Desde luego, y como el mismo Elizaga lo apunta, este cambio "se ve motivado en parte por el envejecimiento relativo de la población, cuando este proceso ocurre".

2 William Petersen escribe: "Los recientes avances en el control de la muerte han sido casi equivalentes a la eliminación de toda mortalidad antes de los 40 años", Population, 1969, p. 575.

3 Jean Bourgeois-Pichat y Chia-lin Pan proponen el uso de "la razón de muertes por enfermedades infecciosas y parasitarias a muertes por todas las causas como un indice de desarrollo de la salud pública" (ello queda incluido en el análisis posterior), "Trends and Determinants of Mortality in Underdeveloped Areas", Trends and Determinants in Mortality, Milbank Memorial Fund, Nueva York, 1956, p. 12.

4 Op. cit., p. 45. 


\section{OBJetivos}

En el presente estudio, de carácter descriptivo más que analítico, se intenta desentrañar un poco el proceso seguido por la mortalidad en México en términos de la mortalidad misma, es decir, de las causas de muerte. El período de estudio es breve, 1950-1967. Sin embargo, en este lapso la mortalidad ha sufrido fuertes cambios: la tasa bruta de mortalidad ha pasado de 17.1 defunciones por mil habitantes en 1949-1951 a 9.5 en 1966-1968.5 En el mismo período la esperanza de vida de la población ha pasado de $49.7^{\circ}$ a, probablemente, un poco más de 62 años. ${ }^{7}$

En realidad el proceso de descenso rápido de la mortalidad se inicia en México aproximadamente hacia el año de 1930 (26.6 por mil es la tasa bruta de mortalidad y 36.9 años la esperanza de vida en 1929-1931), s y hubiera sido lógico estudiar la estructura de la mortalidad por causas a partir de esta fecha; sin embargo, esto se ve dificultado por el tipo de información disponible.

A pesar de las limitaciones es posible esbozar, a grandes rasgos, el proceso de cambio operado en la estructura de la mortalidad en México y efectuar algunas comparaciones con el esquema de evolución de la mortalidad por grupos de causas de defunción -en relación con el nivel de mortalidad general en términos de esperanza de vida al nacimiento - descrito por Naciones Unidas, ${ }^{9}$ e inferir algunas conclusiones, o simplemente exponer los resultados del análisis.

\section{Clasificación ADOPTADa}

Las causas de defunción son diversas y por lo tanto es necesario hacer un agrupamiento de las mismas. Dado que en el presente caso se trata de examinar la evolución de la mortalidad por causas, en relación con la acción de los factores sociales que operan sobre las condiciones generales de vida, parece conveniente adoptar el agrupamiento propuesto por Naciones Unidas. En esa clasificación "se ha optado por agrupar las enfermedades según su comportamiento frente a la acción sanitaria, es decir su mayor o menor resistencia a los progresos médicos y a los programas de salud pública".10

La totalidad de las causas de muerte se concentra en cinco grandes grupos:

Grupo I: Enfermedades infecciosas y parasitarias, gripe, neumonía y bronquitis en menores de cinco años.

5 Secretaría de Industria y Comercio, Dirección General de Estadística, Anuarios estadísticos de los Estados Unidos Mexicanos, México, varios años.

6 Raúl Benítez Zenteno y Gustavo Cabrera Acevedo, Tablas abreviadas de mortalidad de la población de México, 1930, 1940, 1950, 1960, El Colegio de México, 1967.

7 Cálculos preliminares realizados por Miguel Rodríguez, CEED, El Colegio de México, estiman en 61.9 años la esperanza de vida en 1965.

8 Secretaría de Industria y Comercio, op. cit., y Raúl Benítez Z. y Gustàvo Cabrera A., op. cit.

$\rightarrow$ Naciones Unidas, Botetín de población de las Naciones Unidas, No. 6, 1962, Nueva York, 1963, pp. 113-119.

10 Naciones Unidas, op. cit., p. 78 
Grupo II : Cáncer.

Grupo III: Enfermedades cardiovasculares y bronquitis en los mayores de cinco años.

Grupo IV: Muertes violentas.

Grupo V: Las "otras causas" no comprendidas en los grupos anteriores; grupo residual ${ }^{11}$

El carácter exógeno de las causas del grupo I, así como el endógeno de las de los grupos II y III, parecen bien claros. La naturaleza exógena de las defunciones del grupo IV es de tipo diferente a la del grupo I. La división propuesta no está exenta, desde luego, de críticas y puede discutirse si en cada uno de los grupos mencionados se encuentran la totalidad, y exclusivamente, de las causas de muerte que corresponden a los encabezados respectivos. ${ }^{12}$ Se aceptó esta clasificación, además, por razones de comparabilidad con otros países $\mathrm{y}$ estudios semejantes.

En cuanto al agrupamiento de edades, se sigue también el propuesto por Naciones Unidas, ${ }^{13}$ por las razones de comparabilidad ya expuestas.

\section{LIMITACIONES Y CALIDAD DE LA INFORMACIÓN}

Una serie de circunstancias que a continuación se exponen, presentan graves limitaciones al análisis en cuanto a profundidad y alcance. En primer lugar, los criterios de clasificación se han alterado en el tiempo y en el espacio, aspecto que se presenta asociado a y dependiente de la falta de uniformidad en las prácticas de certificación médica en lo que se refiere a la determinación de la causa básica de defunción. Es evidente que en este campo la demografía depende de los avances y cambios en el conocimiento de la etiología. En segundo lugar, la cobertura y la calidad de la información esta-

11 Estos cinco grupos se forman a partir de la lista abreviada de 50 causas -B. Mayor precisión pudo obtenerse con base en la lista intermedia de 150 rubros -A. Naciones Unidas, op. cit., pp. 78-81. Las listas abreviada, intermedia y detallada ( 1000 causas) pueden consultarse en: Organización Mundial de la Salud, Manual de la clasificación estadistica internacional de enfermedades, traumatismos $y$ causas de defunción, Vol. I, Ginebra, 1957.

12 Juan C. Elizaga considera, por ejemplo, endógenas las siguientes causas de muerte: "cáncer y otros tumores (140-239); diabetes mellitus (260); lesiones vasculares que afectan al sistema nervioso central (330-334); enfermedades del sistema circulatorio (400-468); neumonía (490-496); nefritis (590-594); senilidad (794); causas indeterminadas en proporción al total de las muertes por las causas anteriores. La neumonía se consideró causa endógena desde los 25 años de edad y exógena antes". Se indica entre paréntesis el código correspondiente de la Lista Internacional de 1948. Op. cit., p. 51.

13 Razones prácticas y analíticas son la base del agrupamiento de edades escogido: menores de 1 año, 1-4, 5-14, 15-24, 25-44, 45-64, 65-74 y 75 y más años. Naciones Unidas, op. cit., p. 81. Este agrupamiento de edades aprovecha los resultados del estudio "Análisis factorial de las tasas de mortalidad por edad y por sexo: contribución al estudio de las dimensiones de la mortalidad". Ibid., pp. 153-209. 
dística general repercute proporcionalmente en las estadísticas de causas de defunción. ${ }^{14}$ Estas estadísticas, en lo particular, se ven afectadas por la proporción del total de defunciones que cuenta con diagnóstico médico. Esta proporción ha pasado de un $53.1 \%$ en 1950 a un $72 \%$ en 1967. En tercer lugar, en lo que se refiere al aspecto de la comparabilidad, debe considerarse que la estructura misma de la mortalidad por causas es afectada por el nivel mismo de la mortalidad, dependiendo de los momentos históricos diferentes en que se alcanza un nivel dado, de la diversa velocidad con que se da el cambio social y el cambio mismo de la mortalidad, por la aplicación de las técnicas médicas diferentes según la época. De este modo, las observaciones del análisis presentan fuertes limitaciones. Ellas se refieren, por otra parte, al país en su conjunto, dentro del cual existen grandes diferencias. ${ }^{15}$

Se ha propuesto ${ }^{16}$ que la cuantia del rubro B-45 (de la lista abreviada de 50 causas) se tome como indicador de la calidad de las estadísticas de causas de defunción. En este rubro se incluye a las defunciones por causas desconocidas, mal definidas y las atribuidas a la senilidad.

En México, la proporción de este rubro es bastante elevada y su peso aumentó en los últimos años de $11.4 \%$ en 1950 a $18 \%$ en 1967 (en 1930 representó el $15.5 \%$ ). El hecho es un poco extraño si se piensa que en el mismo período el porciento de defunciones con diagnóstico médico se ha incrementado. Cambios en los criterios de diagnóstico o clasificación pudieron originar el ascenso de este rubro.

Por otra parte, "el incremento del peso del rubro B-45, como índice de calidad, en los últimos años, hace pensar en la posibilidad de una mayor cobertura en la declaración de defunción (inclusión de zonas rurales apartadas, etc.) que no se ve acompañada por servicios médicos que mejoren la calidad de la certificación por causas".17

Como se observa en el cuadro 1 , el incremento de la proporción que representan las causas desconocidas, mal declaradas o atribuidas a senilidad se manifiesta en todos los grupos de edad, con excepción de los menores de un año, proporción que alcanza en 1967 un $20 \%$ y más del total de defunciones en casi todas las edades, lo que indudablemente representa una importante limitación en el análisis de la estructura de la mortalidad por causas.

14 Hubiera sido conveniente, a fin de evitar la influencia de las variaciones aleatorias del fenómeno en estudio, haber tomado promedios de tres años consecutivos. Limitaciones de tiempo no lo permitieron.

$15 \mathrm{Se}$ encuentra en preparación un estudio a nivel regional, para 1960, por Ricardo Alvarado.

16 Naciones Unidas, op. cit., p. 75.

17 Ricardo Alvarado y pFrancisco Alba H., "México: estudio de la mortalidad por causas, ambos sexos, 1930, 1950, 1956, 1960", ponencia presentada a la Conferencia Regional Latinoamericana de Población, México, agosto de 1970.

18 En algunos estudios se suele distribuir la proporción del rubro B-45 entre los grupos de causas para obviar este inconveniente. En el presente caso "se optó por no distribuir el rubro B-45 entre los grupos de causas por parecer arbitrario cualquier método de distribución, dado que la magnitud y el significado de este rubro han cambiado sustancialmente en México a través del tiempo (1930-1967)". Alvarado y Alba, op. cit. 


\section{Cuadro 1}

México: Defunciones inclutdas en el Rubro B-45, POR GRÚpos De edad Y PARA AMBOS SEXOS, 1950-1967

(Porcientos)

\begin{tabular}{crrrrr}
\hline $\begin{array}{c}\text { Grupos de } \\
\text { edad }\end{array}$ & $1950^{\mathrm{a}} /$ & $1955^{\mathrm{a}}$ & $1960 \mathrm{~b}$ & $1965^{\mathrm{b}} /$ & $1967^{\mathrm{b}} /$ \\
\hline Todas Ias edades & 11.42 & 9.85 & 12.14 & 18.62 & 17.91 \\
0 & 4.34 & - & 1.82 & 1.40 & 1.29 \\
$1-4$ & 8.31 & 8.12 & 12.08 & 21.07 & 20.23 \\
$5-14$ & 11.11 & 10.66 & 14.32 & 24.54 & 24.16 \\
$15-24$ & 9.21 & 8.60 & 11.14 & 18.43 & 18.97 \\
$25-44$ & 8.53 & 8.47 & 10.36 & 17.69 & 17.72 \\
$45-64$ & 9.93 & 9.79 & 10.31 & 20.05 & 20.10 \\
$65-74$ & 18.88 & 17.75 & 29.48 & 27.35 & 25.92 \\
75 y mas & 39.82 & 36.94 & & 42.83 & 41.91 \\
\hline
\end{tabular}

Fuentes: a Secretaría de Industria y Comercio, Dirección General de Estadística Defunciones por causas, mecanografiado.

b Secretaría de Salubridad y Asistencia, Dirección de Bioestadística, Estadisticas vitales de los Estados Unidos Mexicanos.

\section{Estructura DE La MORTALIDAd POR CAUSAS}

De la observación del cuadro 2 resulta claro que ha sido el progresivo control de las enfermedades infecciosas y parasitarias (grupo I) a lo que, básicamente, se debe el descenso de la mortalidad en México. La tasa de mortalidad por este tipo de causas, 218 por cien mil en 1967, es un quinto de lo que fue en 1930 -1 136 por cien mily casi un tercio de la prevaleciente en 1950. Las defunciones de tipo infeccioso y parasitario, son causantes principales no sólo del descenso experimentado por la mortalidad sino del nivel de la misma; ya que, si bien la proporción de las defunciones del grupo $I$ ha disminuido en el tiempo ( $42.6 \%$ en 1930 y $23.9 \%$ en 1967), una parte muy importante de las defunciones del grupo residual $\mathrm{V}$ debe atribuirse a esta clase de causas de muerte.

\section{Cuadro 2}

MÉxico: Mortalidad POR CaUSas, ambos SeXos, 1930, 1950 y 1967

\begin{tabular}{|c|c|c|c|c|c|c|c|c|}
\hline \multirow{2}{*}{$\begin{array}{l}\text { Grupos do } \\
\text { caugas do } \\
\text { defuncisn }\end{array}$} & \multicolumn{3}{|c|}{$\begin{array}{c}\text { Tasas de mortalidad } \\
(\operatorname{por} 100000)\end{array}$} & \multicolumn{2}{|c|}{ Cambios porcentuales } & \multicolumn{3}{|c|}{$\begin{array}{c}\text { Distribucion de las defunciones } \\
\text { (poreientos) }\end{array}$} \\
\hline & $1930^{\mathrm{e} /}$ & $1950^{b / 2}$ & $1967^{\text {c/ }}$ & $\begin{array}{l}1930-1967 \\
(1930=100)\end{array}$ & $\begin{array}{l}1950-1967 \\
(1950=100)\end{array}$ & $1930^{8 / 2}$ & $1950^{b /}$ & 1967 ef \\
\hline $\begin{array}{l}\text { Todas } \\
\text { as causa } \\
\text { I } \\
\text { II } \\
\text { III } \\
\text { IV } \\
\text { V } \\
\end{array}$ & $\begin{array}{rr}2667 \\
1136 \\
& 15 \\
& 82 \\
& 110 \\
1 & 324 \\
\end{array}$ & $\begin{array}{r}1615 \\
580 \\
29 \\
94 \\
94 \\
818 \\
8 \\
\end{array}$ & $\begin{array}{r}911 \\
218 \\
34 \\
63 \\
65 \\
531 \\
\end{array}$ & $\begin{array}{l}-65.84 \\
-80.81 \\
+126.67 \\
-23.17 \\
-40.91 \\
-59.89\end{array}$ & $\begin{array}{l}-43.59 \\
-62.41 \\
+17.24 \\
-32.98 \\
-30.85 \\
-35.09 \\
\end{array}$ & $\begin{array}{r}100.00 \\
42.60 \\
.54 \\
3.09 \\
4.14 \\
49.63 \\
\end{array}$ & $\begin{array}{r}100.00 \\
35.93 \\
1.78 \\
5.84 \\
5.83 \\
50.62 \\
\end{array}$ & $\begin{array}{r}100.00 \\
23.91 \\
3.74 \\
6.87 \\
7.15 \\
58.33 \\
\end{array}$ \\
\hline
\end{tabular}

Fuentes: a Elaborado con base en información recolectada por Eduardo Cordero, CEED, El Colegio de México.

b Secretaría de Industria y Comercio, op. cit.

c Secretaría de Salubridad y Asistencia, op. cit. 
Los cambios ocurridos en los demás grupos de causas, sin juzgarlos despreciables, no parece que sean determinantes en el comportamiento de la mortalidad general. Los niveles de sus tasas son todavía muy pequeños en relación con los niveles de los grupos I y V, y sus cambios son también comparativamente menores. Su participación dentro del total de causas, aunque se ha ido incrementando, es relativamente pequeña; las causas de origen "degenerativo" (grupos II y III) pasan de un $3.6 \%$ a un $10.6 \%$, y las muertes violentas de un $4.1 \%$ a un $7.15 \%$, entre 1930 y 1967, respectivamente. Parece pues que en México la mortalidad exógena ha sido y sigue siendo, a pesar de los avances logrados en el campo de la salud, el principal componente de la mortalidad general.

La apreciación anterior, válida en términos generales, debe matizarse por dos consideraciones: en primer lugar, el número de defunciones clasificadas en el grupo $\mathrm{V}$ va de un 50 a un $60 \%$ del total de defunciones; en segundo lugar, la población ha experimentado en el período un rejuvenecimiento 9

Una forma indirecta de confirmar las anteriores observaciones es examinar la estructura de la mortalidad por grupos de edad. Se sabe que las enfermedades de origen infeccioso y parasitario atacan, de manera principal a los grupos de edades bajas. Las enfermedades de tipo endógeno - "degenerativas del organismo"- son propias, en cambio, de las edades avanzadas. En el cuadro 3 se presentan las distribuciones de las defunciones por grupos de edad en varios años entre 1950 y 1967. Se observa en el cuadro que la estructura de la mortalidad sólo ha sufrido ligeros cambios: las defunciones de los menores de 5 años han descendido de un $48 \%$ en 1950 a un $43.5 \%$ en 1967, descenso que se debe exclusivamente a una menor contribución de las defunciones ocurridas entre las edades de 1 a 4 años, ya que las defunciones de los menores de un año aun se han incrementado. Las defunciones de personas mayores de 65 años tienen mayor importancia en el transcurso del tiempo, cambios que, sin

\section{Cuadro 3}

México: Distribución de las defunctones por grupos de EDad, ambos SeXos, 1950-1967

\begin{tabular}{|c|c|c|c|c|c|}
\hline \multirow{2}{*}{$\begin{array}{l}\text { Grupos de } \\
\text { edades }\end{array}$} & \multicolumn{3}{|c|}{ Distribueisn } & \multicolumn{2}{|c|}{ porcentug 1} \\
\hline & $1950^{8 / 7}$ & $1955^{\mathrm{a} /}$ & $1960^{b / 2}$ & $1965^{\mathrm{b} /}$ & 1967 b? \\
\hline $\begin{array}{c}\text { Todas las edades } \\
0 \\
1-4 \\
5-14 \\
15=24 \\
25=44 \\
45=64 \\
65=74 \\
75 \text { y má }\end{array}$ & $\begin{array}{r}100.00 \\
27.04 \\
21.02 \\
5.72 \\
5.13 \\
11.66 \\
12.83 \\
7.05 \\
9.52\end{array}$ & $\begin{array}{r}100.00 \\
28.19 \\
21.48 \\
5.65 \\
4: 66 \\
10.43 \\
12.27 \\
7.19 \\
10.13\end{array}$ & $\begin{array}{r}100.00 \\
29.68 \\
16.70 \\
5.07 \\
4.43 \\
10.64 \\
13.61 \\
19.87\end{array}$ & $\begin{array}{r}100.00 \\
28.36 \\
14.67 \\
4.99 \\
4.30 \\
10.86 \\
14.04 \\
8.91 \\
13.87\end{array}$ & $\begin{array}{r}100.00 \\
29.74 \\
13.84 \\
4.74 \\
4.26 \\
10.82 \\
13.76 \\
9.27 \\
13.57\end{array}$ \\
\hline
\end{tabular}

Fuentes: a Secretaría de Industria y Comercio, op. cit.

b Secretaria de Salubridad y Asistencia, op. cit.

19 La población menor de 15 años ha pasado de un $39.2 \%$ en 1930 a un $46.2 \%$ en 1970 (según datos preliminares). 
Cuadro 4

TASAS COMPARATIVAS DE MORTALIDAd POR GRUPOS DE CAUSAS Y DISTRIbUCión DE LAS DEFUNCIONES Países y años seleccionados

\begin{tabular}{|c|c|c|c|c|c|c|c|c|c|c|c|c|c|c|}
\hline \multirow{2}{*}{ PaIs $y$ año } & \multirow{2}{*}{$\begin{array}{c}\theta_{0} \\
(a \tilde{n} 08)\end{array}$} & \multirow{2}{*}{$\begin{array}{c}\text { Rubro } \\
B-45 \\
(\%)\end{array}$} & \multicolumn{6}{|c|}{$\begin{array}{r}\text { Tasas comparativas } \\
\text { (por } 100000)\end{array}$} & \multicolumn{6}{|c|}{ Distribucion porcentual } \\
\hline & & & Total & $I$ & II & III & IV & $\overline{\mathrm{V}}$ & Total & I & II & III & IV & $\bar{v}$ \\
\hline Japbn, 1935-1936 & 48.3 & 9.9 & 1752 & 500 & 70 & 252 & 65 & 865 & 100.0 & 28.5 & 4.3 & 14.4 & 3.7 & 49.4 \\
\hline M6xico, $1950^{\mathrm{b} /}$ & 49.7 & 11.4. & 1702 & 589 & 35 & 114 & 99 & 865 & 100.0 & 34.6 & 2.1 & 6.7 & 5.8 & 50.8 \\
\hline $\begin{array}{c}\text { Inglaterra y Galas, } \\
1901-1910 \mathrm{~g} /\end{array}$ & 50.5 & 5.7 & 1620 & 511 & 83 & 358 & 59 & 608 & 100.0 & .31 .6 & 5.2 & 22.1 & 3.6 & 37.5 \\
\hline $\begin{array}{c}\text { Inglaterra y Galad } \\
1911-1920 \mathrm{a}\end{array}$ & 55.0 & 5.2 & 1448 & 472 & 91 & 297 & 61 & 528 & 100.0 & 32.6 & 6.3 & 20.5 & 4.2 & 36.4 \\
\hline Mexico, $1956^{\mathfrak{b} /}$ & 56.5 & 11.0 & 1271 & 353 & 40 & 124 & 89 & 665 & 100.0 & 27.8 & 3.1 & 9.8 & 7.0 & 52.3 \\
\hline Méxioo, $1960^{\mathrm{b}}$ / & 58.9 & 12.1 & 1211 & 311 & 45 & 123 & 82 & 650 & 100.0 & 25.7 & 3.7 & 10.1 & 6.8 & 53.7 \\
\hline $\begin{array}{c}\text { Inglaterra } y \text { Galos, } \\
1921-1930 \mathrm{a} /\end{array}$ & 59.0 & 4.1 & 1109 & 321 & 105 & 302 & 46 & 336 & 100.0 & 28.9 & 9.5 & 27.2 & 4.1 & 30.3 \\
\hline Mexico, 1967 b/ & 63.0 & 17.9 & 935 & 199 & 44 & 81 & 73 & $538^{\circ}$ & 100.0 & 21.3 & 4.7 & 8.7 & 7.8 & 57.5 \\
\hline $\begin{array}{c}\text { Costa Rica, } \\
1962-1964 \text { d }\end{array}$ & 63.3 & 12.6 & 856 & 152 & 101 & 128 & 46 & 429 & 100.0 & 17.8 & 11.8 & 15.0 & 5.4 & 50.0 \\
\hline Japón, 1951-1953 & 63.5 & 9.9 & 925 & 193 & 76 & 205 & 59 & 391 & 100.0 & 20.9 & 8.2 & 22.2 & 6.4 & 42.3 \\
\hline $\begin{array}{l}\text { Dinamarca, } \\
1936-1938^{\text {a/ }}\end{array}$ & 64.5 & 5.1 & 869 & 188 & 100 & 186 & 45 & 350 & 100.0 & 21.6 & 11.5 & 21.4 & 5.2 & 40.3 \\
\hline
\end{tabular}

Fuentes: a Naciones Unidas, Boletín de población de las Naciones Unidas, No. 6, 1962, cuadro V. 32, p. 114.

b Elaboración de los autores. Regional Latinoamericana de Población, México, agosto de 1970. 
embargo, no son lo suficientemente decisivos como para afirmar que la estructura de la mortalidad por edades se haya alterado sensiblemente.

La evolución de las defunciones por grupos de causas en México ${ }^{20}$ concuerda, en sus rasgos generales, con la evolución histórica de la mortalidad por causas. Este hecho puede examinarse de dos maneras: comparando la estructura de la mortalidad por causas de México con las de otros países, para esperanzas de vida al nacimiento similares, y con el esquema de evolución de la mortalidad por grandes grupos de causas elaborado por Naciones Unidas. ${ }^{21}$

En el cuadro 4 aparecen las tasas comparativas y la distribución de las muertes por grupos de causas en México y algunos países seleccionados. Se observa en él que las tasas de mortalidad por cáncer (grupo II) son sistemáticamente menores en México, a la mitad de los niveles de los demás países (alrededor de 40 por cien mil en México contra valores de 80-100 en el resto de los países incluidos en el cuadro); que el mismo caso se presenta respecto al grupo de causas de muerte de origen cardiovascular (III), pues las tasas correspondientes de México están a un tercio o a la mitad de las tasas de los otros países. Puede ser significativo que la tasa de mortalidad por este tipo de causas en Costa Rica sea sólo un poco superior a la de México: 128 por cien mil corresponde a Costa Rica para una esperanza de vida al nacer de 63.5 años; a México, 81 cuando la esperanza de vida aproximada es de 63 años, pero 125 por cien mil cuando su esperanza de vida se situaba entre los 56 y los 59 años. En el grupo de muertes violentas (IV), las tasas en México son más elevadas, hasta en un $50 \%$ con tendencias a una menor diferenciación.

La secuencia descendente de la mortalidad del grupo de causas infecciosas y parasitarias no muestra "desviaciones" respecto al comportamiento en los demás países. El grupo residual (V), en cambio, desciende más lentamente y las tasas respectivas presentan discrepancias apreciables cada vez mayores respecto a las menores tasas de los atros países. El cuadro muestra la participación que tiene el rubro B-45 en el total de causas, por parecer que las diferencias aquí presentes son, en parte, causantes de algunas de las diferencias observadas en las tasas comparativas $\mathrm{y}$, en consecuencia, también de las diferencias que pudieran observarse en las distribuciones porcentuales de las defunciones de México y los otros países considerados, semejanzas y discrepancias similares a las observadas respecto a las tasas comparadas.

Un elemento de distorsión en la comparación son las diferentes épocas en que los países alcanzan determinados niveles de mortalidad, y las técnicas empleadas. A este hecho puede deberse que los países que más recientemente han alcanzado niveles bajos de mortalidad tienden a presentar tasas de mortalidad por enfermedades infecciosas y parasitarias un tanto menores a las que les correspon-

20 Para ello se obtuvieron tasas comparativas de mortalidad por grupos de causas, con base en una población tipo: la mundial a mediados de 1960 . Ver Naciones Unidas, op. cit., p. 114.

21 Naciones Unidas, op. cit., pp. 113-119. El esquema seguido es el de una población con estructura de edades "joven". 
dieron a pueblos que alcanzaron los mismos niveles de mortalidad con anterioridad. En las distribuciones de las defunciones se refleja lo apuntado: México -311 y $25.7 \%$ en 1960 - e Inglaterra y Gales -321 y $28.9 \%$ durante 1921-1930 - a un nivel de 59 años de esperanza de vida, al igual que Costa Rica -152 y $17.8 \%$ durante 1962-1964- y Dinamarca -188 y $21.6 \%$ durante 1936-1938- a un nivel de 63-64 años de esperanza de vida.

Con la intención de apuntar lo que pudiera considerarse rasgos característicos de la estructura de la mortalidad en México, se incluye una comparación con el esquema de evolución de la mortalidad de las Naciones Unidas (el esquema no se considera "modelo", sino mera aproximación o indicador). El cuadro 5 ilustra este aspecto.

A México le correspondería, dada la época que se estudia, la segunda mitad de la primera etapa de la evolución (a partir de los 50 años de esperanza de vida): "cuando la expectativa de vida al nacer pasa de 40 a 60 años la proporción de las muertes debidas a las enfermedades del grupo I (enfermedades infecciosas y parasitarias, gripe, pulmonía) disminuye. La proporción de las muertes debidas a las enfermedades de los grupos II (cáncer) y III (enfermedades cardiovasculares y bronquitis después de los 5 años) aumenta. La proporción de las defunciones debidas a las enfermedades del grupo V (otras enfermedades) aumenta ligeramente".22 Asimismo, corresponde a México la primera parte de la segunda etapa, "cuando la esperanza de vida al nacer pasa de 60 a 70 años, la parte de las enfermedades del grupo I continúa disminuyendo, y la de las enfermedades de los grupos II y III sigue aumentando pero el ritmo de aumento se acelera. En cuanto a la parte correspondiente a las enfermedades del grupo $\mathrm{V}$, permanece prácticamente invariable".23

La evolución de la estructura de la mortalidad por grandes grupos de causas en México se inserta, globalmente, en la evolución general descrita; se aparta de ella en los mismos puntos ya mencionados en la comparación con otros países: la menor importancia de las enfermedades "degenerativas" de los grupos II y III, y la mayor del grupo de muertes violentas. Es clara la discrepancia presente en cuanto al grupo V de "otras causas"; hecho que convierte en problemática la comparación. En cambio, queda fuera de duda el rápido descenso de la mortalidad del grupo I, y en menor medida, el de los grupos IV y V.

En términos generales, el comportamiento por sexo no difiere def comportamiento conjunto. Se advierte en el cuadro 6 que la mortalidad femenina supera a la masculina en los grupos de causas "degenerativas" del propio organismo (II y III); esto es perceptible claramente en 1950 y 1960 , pero tiende a diluirse en fechas recientes, principalmente en lo que se refiere al grupo III. ${ }^{24}$ La mayor mortalidad masculina por muertes violentas (grupo IV) es una de las ca-

22 Naciones Unidas, op. cit., p. 116.

23 Ibid., pp. 116-117.

24 Se realizaron cálculos para 1955 y 1965 a fin de detectar posibles com. portamientos extraños dentro de la evolución descrita; no se encontró cambio algumo que no esté presente en los años que se examinan. Los datos se incluyen en el apéndice. 


\section{Cuadro 5}

TaSas COMPaRativas de MORTALIDAD POR CAUSAS Y DISTRIBUCIÓN DE LAS DEFUNCIONES

\begin{tabular}{|c|c|c|c|c|c|c|c|c|c|c|c|c|c|}
\hline & \multirow{2}{*}{$\therefore$} & \multicolumn{6}{|c|}{$\begin{array}{c}\text { Tasas comparativas } \\
(\text { por } 100000)\end{array}$} & \multicolumn{6}{|c|}{ Distribuciones porcentuales } \\
\hline & & Total & I & II & III & IV & $\mathrm{v}$ & Total & I & II & III & IV & $\mathrm{v}$ \\
\hline México $(1950) \stackrel{a}{a}$ & 49.7 & 1702 & 589 & 35 & 114 & 99 & 865 & 100.0 & 34.6 & 2.1 & E.7 & 5.8 & 50.8 \\
\hline Esquema N.U. b/ & 50.0 & 1629 & 556 & 91 & 304 & 70 & 608 & 100.0 & 34.1 & 5.6 & 18.7 & 4.3 & 37.3 \\
\hline Esquema N.U. b/ & 56.0 & 1278 & 360 & 91 & 271 & 62 & 494 & 100.0 & 28.2 & 7.1 & 21.2 & 4.9 & 38.7 \\
\hline México (1956) a & $(56.5)$ & 1271 & 353 & 40 & 124 & 89 & 665 & 100.0 & 27.8 & 3.1 & 9.8 & 7.0 & 52.3 \\
\hline Esquema N.U. b/ & 58.0 & 1174 & 304 & 91 & 260 & 59 & 460 & 100.0 & 25.9 & 7.8 & 22.1 & 5.0 & 39.2 \\
\hline Mêxico (1960) $\mathfrak{a} /$ & 58.9 & 1211 & 311 & 45 & 123 & 82 & 650 & 100.0 & 25.7 & 3.7 & 10.1 & 6.8 & 53.7 \\
\hline Esquema N.u. b/ & 60.0 & 1072 & 254 & 91 & 249 & 56 & 422 & 100.0 & 23.7 & 8.5 & 23.2 & 5.2 & 39.4 \\
\hline Esquema N.U. $b /$ & 62.0 & 972 & 208 & 91 & 238 & 53 & 382 & 100.0 & 21.4 & 9.4 & 24.5 & 5.5 & $39 \cdot 3$ \\
\hline México (1967) a/ & $(63.0)$ & 935 & 199 & 44 & 81 & 73 & 538 & 100.0 & 21.3 & 4.7 & 8.7 & 7.8 & 57.5 \\
\hline Esquema N.U. b/ & $(64 \cdot 0)$ & 876 & 166 & 91 & 227 & 50 & 342 & 100.0 & 18.9 & 10.4 & 25.9 & 5.7 & 39.0 \\
\hline
\end{tabular}

Fuentes: a Elaboración de los autores.

b Naciones Unidas, op. cit., cuadro V. 33, p. 116. 
racterísticas diferenciales más marcadas. El fenómeno, por otra parte, es universal. El cambio en la actividad y comportamiento de la mujer dentro de una sociedad moderna tiende a reducir este diferencial. ${ }^{25}$ Esta reducción es bien sensible en México entre 1950 y 1967 ; de ser 5 veces superior el nivel de la mortalidad masculina en la primera de estas fechas, al de la femenina, se reduce a 4 veces en la última fecha, debido a un descenso más pronunciado de la mortalidad por causas violentas entre el sexo masculino - de 159 a 104 por cien milque entre el femenino - de 31 a 26 - en el período considerado.

\section{Cuadro 6}

México: Mortalidad POR gRupos de causas de defunctón y por SEXo, 1950,1960 Y 1967

\begin{tabular}{|c|c|c|c|c|c|c|}
\hline & \multicolumn{6}{|c|}{ Grupos de causas de defunción } \\
\hline & $\begin{array}{c}\text { Todas las } \\
\text { causas }\end{array}$ & I & II & III & IV & v. \\
\hline & \multicolumn{6}{|c|}{ Pasas de mortalidad (por 100 000) } \\
\hline 1250 & & & & & & \\
\hline $\begin{array}{l}\text { Hombres } \\
\text { Mujeres }\end{array}$ & $\begin{array}{l}1717 \\
17 \\
517\end{array}$ & 608 & 21 & 89 & 159 & 840 \\
\hline $\begin{array}{c}\text { Mujeres } \\
1960\end{array}$ & 1517 & 553 & 37 & 99 & 31 & 797 \\
\hline $\begin{array}{l}\text { Hombres } \\
\text { Mujeres }\end{array}$ & $\begin{array}{l}1216 \\
1083\end{array}$ & $\begin{array}{l}328 \\
300\end{array}$ & $\begin{array}{l}28 \\
44\end{array}$ & $\begin{array}{r}92 \\
103\end{array}$ & $\begin{array}{r}121 \\
29\end{array}$ & $\begin{array}{l}647 \\
607\end{array}$ \\
\hline \multicolumn{7}{|c|}{1967} \\
\hline $\begin{array}{l}\text { Hombres } \\
\text { Mujeres }\end{array}$ & $\begin{array}{l}969 \\
853\end{array}$ & $\begin{array}{l}228 \\
208\end{array}$ & $\begin{array}{l}28 \\
40\end{array}$ & $\begin{array}{l}61 \\
64\end{array}$ & $\begin{array}{r}104 \\
26\end{array}$ & $\begin{array}{l}548 \\
515\end{array}$ \\
\hline & \multicolumn{6}{|c|}{ Distribución do las defunciones ( $\%$ ) } \\
\hline 195 & & & & & & \\
\hline $\begin{array}{l}\text { Hombres } \\
\text { Mujeres }\end{array}$ & $\begin{array}{l}100.00 \\
100.00\end{array}$ & $\begin{array}{l}35.44 \\
36.48\end{array}$ & $\begin{array}{l}1.20 \\
2.41\end{array}$ & $\begin{array}{l}5.21 \\
6.53\end{array}$ & $\begin{array}{l}9.25 \\
2.07\end{array}$ & $\begin{array}{l}48.90 \\
52.51\end{array}$ \\
\hline 1960 & & & & & & \\
\hline $\begin{array}{l}\text { Hombres } \\
\text { Mujeres }\end{array}$ & $\begin{array}{l}100.00 \\
100.00\end{array}$ & $\begin{array}{l}26.94 \\
27.72\end{array}$ & $\begin{array}{l}2.30 \\
4.02\end{array}$ & $\begin{array}{l}7.59 \\
9.50\end{array}$ & $\begin{array}{l}9.94 \\
2.65\end{array}$ & $\begin{array}{l}53.23 \\
56.11\end{array}$ \\
\hline 1967 & & & & & & \\
\hline $\begin{array}{l}\text { Hombres } \\
\text { Mujeres }\end{array}$ & $\begin{array}{l}100.00 \\
100.00\end{array}$ & $\begin{array}{l}23.47 \\
24.41\end{array}$ & $\begin{array}{l}2.91 \\
4.69\end{array}$ & $\begin{array}{l}6.33 \\
7.48\end{array}$ & $\begin{array}{r}10.74 \\
3.04\end{array}$ & $\begin{array}{l}56.55 \\
60.38\end{array}$ \\
\hline
\end{tabular}

Se carece, en realidad, de más información a fin de analizar adecuadamente el comportamiento diferencial entre los sexos. La experiencia de algunos países de baja mortalidad sugiere que con el descenso de la mortalidad general la sobremortalidad femenina en los grupos II y III tiende a desaparecer e incluso se invierte. ${ }^{26}$ En México, aunque sus tasas se encuentran a un nivel relativo bajo, la tendencia a la nivelación de las tasas por sexo es ya perceptible.

Con base en estas consideraciones y observando también que la mortalidad del sexo masculino es mayor que la del femenino en los grupos I y V, es claro que el fenómeno de la sobremortalidad masculina se acentúe con los bajos niveles y sea extensivo a todas las edades: puesto que lo es, o tiende a serlo, en todos los grupos de causas.

25 Naciones Unidas, op. cit., p. 103.

26 Ibid., op. cit., cuadros de las pp. 93 y 99. 
Gráfica 1

México : Tasas de MorTalidad por CaUSas, ambos SeXos, 1950 Y 1957

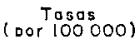

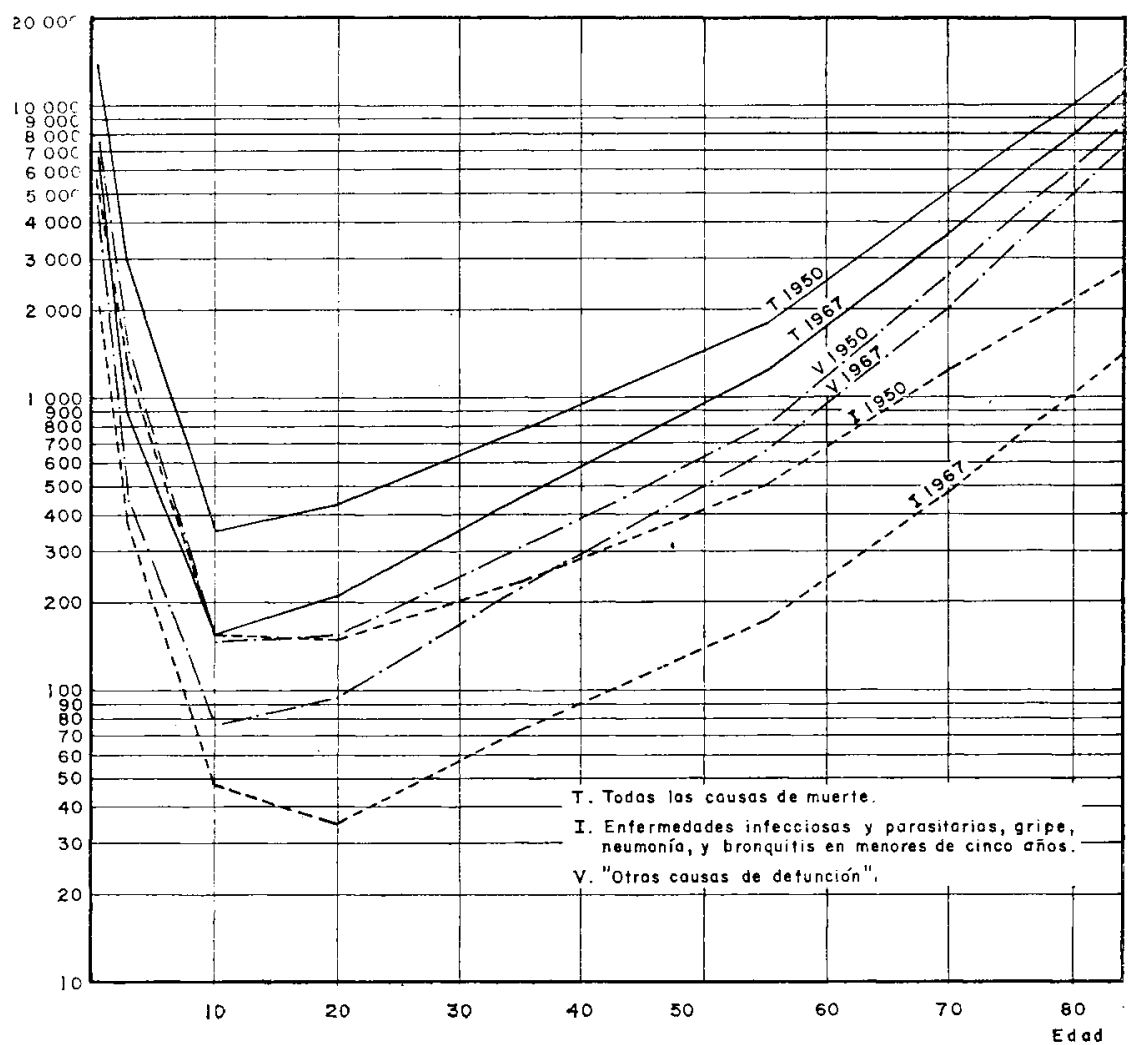

La estructura de la mortalidad de cada sexo resulta afectada, en consecuencia, por estas diferencias en la mortalidad por grupos de causas. Así, el grupo de muertes violentas -éstas han aumentado su participación relativa- oscila entre 9 y $11 \%$ del total de defunciones masculinas, y sólo entre 2 y $3 \%$ de las femeninas. La proporción de las defunciones de los grupos II y III es de un $12 \%$ en el sexo femenino y un $9 \%$ en el masculino, en 1967.$]$

Se examinan a continuación los cinco grupos de causas de defunción en relación con su comportamiento dentro de grupos de edad seleccionados. El análisis no puede ser ni profundo ni detallado pues en todos los grupos de edad, excepto los menores de un año, la importancia de las defunciones por causa mal definida, desconocida $o$ atribuida a senilidad (rubro B-45) es muy elevada, como se observa en el cuadro 1, lo que implica una gran limitación.

Las gráficas 1 y 2 ilustran los rasgos que se describen..$^{27}$

27 En el apéndice se presenta la información estadística (detallada y para varios años). 
Gráfica 2

México: Tasas de mortalidad por causas, ambos SEXos, 1950 y 1967

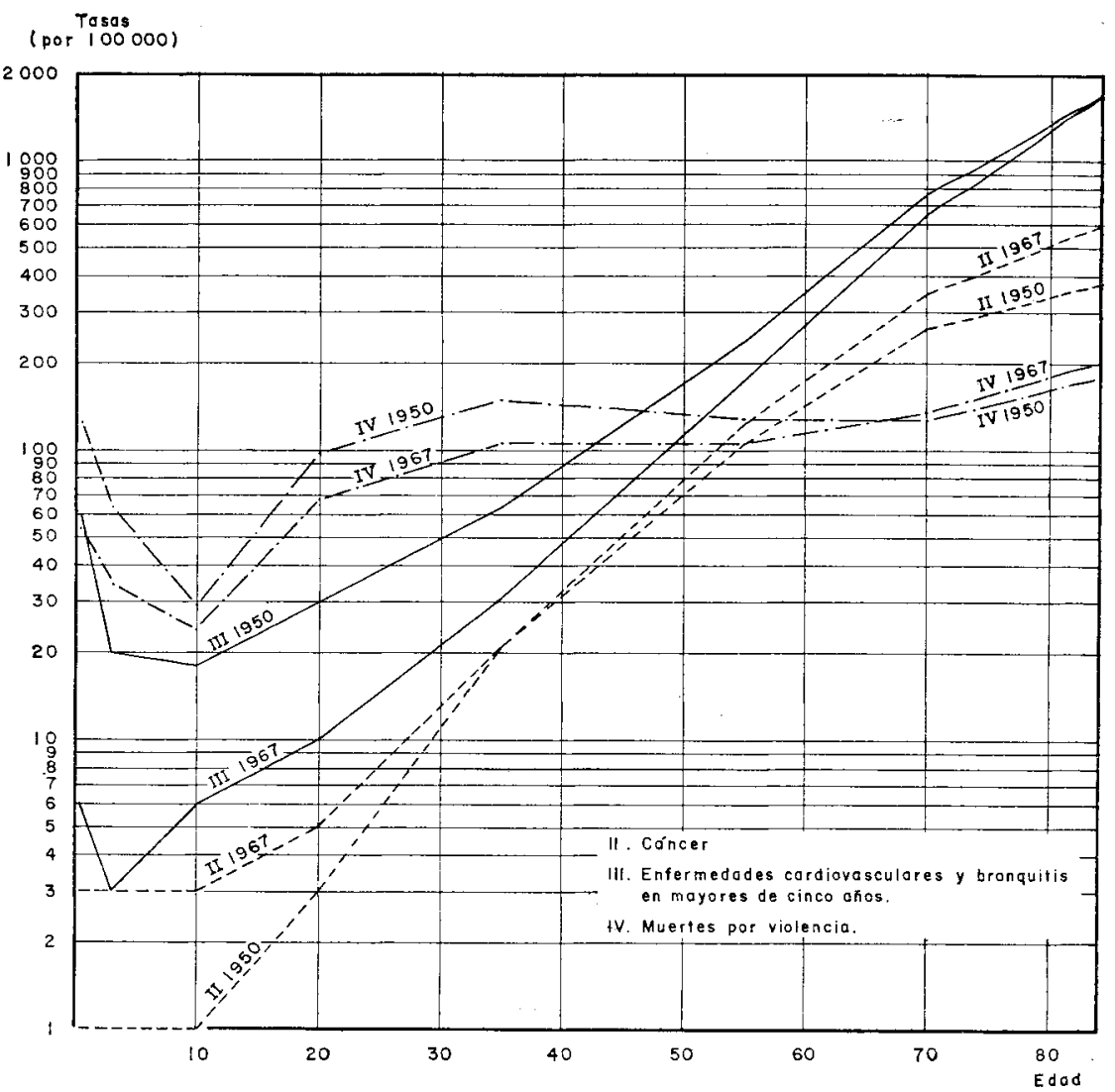

1. Mortalidad por causas infecciosas y parasitarias, gripe, neumonía $y$ bronquitis en menores de cinco años

La mortalidad del grupo I experimenta los mayores descensos en todos los grupos de edad entre 1950 y 1967 . Entre los 15 y los 24 años esta disminución adquiere su mayor intensidad; en más de un $75 \%$ disminuye la mortalidad por este tipo de causas y para este grupo de edades en el período que se considera; el descenso es de alrededor de un $70 \%$ en el resto de las edades, entre 1 y 44 años.

No obstante la magnitud de estos descensos, los niveles alcanzados no pueden considerarse satisfactorios, pues el nivel de mortalidad del grupo I, para todas las edades en conjunto, era en México en 1967 siete veces superior al alcanzado por Holanda en 1954-1956.28 Todavía

28 Las tasas respectivas son 199 y 28 por cien mil. Véanse el cuadro 4 y el cuadro V. 32 de Naciones Unidas, op. cit., p. 114. 
en 1967, el $43.5 \%$ de las defunciones ocurridas entre 1 y 5 años correspondía a estas causas; este porciento varía para los grupos restantes de edad y todavía alcanza alrededor de un $13 \%$ en los mayores de 65 años. Por otra parte, existen a la vez indudables indicios de mejoría: por ejemplo, en el grupo de 15-24 años, el porciento de este tipo de causas de muerte descendió de $34.7 \%$ en 1950 a $16.6 \%$ en 1967.

En cuanto a sexo, se advierte que la mortalidad masculina es superior en los menores de un año $\mathrm{y}$, generalmente, a partir de los 25 años; al final de la vida a veces acontece lo inverso. El descenso experimentado en el período ha favorecido al sexo femenino entre los 25 y los 74 años y al masculino entre los 15 y los 24 años, siendo semejante el descenso en las edades restantes.

Las enfermedades infecciosas y parasitarias, propias de las primeras edades, aún inciden fuertemente en México en éstas y las restantes edades: el $58 \%$ de este tipo de defunciones lo registraban en 1950 los menores de 5 años y el $63.5 \%$ en 1967 . No se notan particularidades al respecto entre los sexos.

\section{Mortalidad por cáncer}

AI examinar este grupo de causas de defunción se debe tener en cuenta, de una manera especial, el problema de la información estadística, ya que este tipo de causas de defunción se ve particularmente afectado por los cambios en los conceptos nosológicos, por los progresos en el diagnóstico médico, etc. ${ }^{29}$

Con frecuencia se habla de que la mortalidad por cáncer ha aumentado, cuando sólo se trata de una mayor proporción de este tipo de muertes respecto al total, dada una significativa disminución de la mortalidad en otras causas distintas al cáncer. Se sabe, por otra parte, que el cáncer incide en mayor medida con el aumento de la edad; cuando las poblaciones envejecen, es lógico que el cáncer aumente en importancia relativa, como causa de muerte.

A pesar de lo anterior, se apuntan algunos rasgos de las estadísticas de mortalidad por cáncer en el período 1950-1967 en México.

Puesto que la mayor parte de las defunciones por cáncer se presenta en edades adultas y avanzadas ( $80 \%$ a partir de los 45 años; $93 \%$ a partir de los 25 , en 1967) y que la mortalidad por cáncer es muy baja en la infancia y edades jóvenes, conviene limitarse a las edades mayores de 24 años. Ligeros cambios se presentan a lo largo del período considerado que son difíciles de atribuir a cambios reales en el comportamiento de la mortalidad por cáncer, por las razones ya mencionadas. Las tasas, en general, han aumentado con intensidad

29 Naciones Unidas, op. cit., p. 90. “... parece posible afirmar que las estadísticas de causas de fallecimiento tal como se establecen actualmente no constituyen una herramienta de análisis suficientemente aguda como para clescubrir las variaciones en la mortalidad por cáncer. Conviene señalar, sin embargo, que las imperfecciones de las estadísticas sobre causas de fallecimiento no se limitan únicamente al cáncer. Existen en todas las causas y el hecho de que las estadísticas no puedan medir las variaciones de la mortalidad por cáncer sólo significa que estas variaciones son débiles en comparación con las modificaciones sufridas por las otras causas de fallecimiento que sí se leen claramente en las cifras publicadas..." Ibid., pp. 93-95. 
variable según los grupos de edad; la única excepción es un descenso, entre 1950 y 1967, de un $6.5 \%$ en el grupo de mujeres de 25 a 44 años. El incremento de la mortalidad por cáncer es mayor conforme la edad es más avanzada: alcanza un $60 \%$ en el grupo de 75 años y más.

Esta mayor mortalidad por cáncer alcanza con más intensidad a los hombres que a las mujeres. Así, en las edades 65-74 años, el incremento es de 48.5 y $19.5 \%$, respectivamente. Con ello se suprimen o acortan los diferenciales en la mortalidad por cáncer (para el grupo de edades 45-64 años, las tasas de mortalidad por cáncer fueron, en 1950 , de 70 y 140 por cien $\mathrm{mil}$; en 1967, de 98 y 149, respectivamente, del sexo masculino y femenino).

Con el aumento de la mortalidad por cáncer y la disminución de la misma por otras causas, la participación relativa, general y en los grupos de edades, ha ido aumentando paulatinamente; en algunos grupos de edad casi se ha duplicado, como en el grupo 45-64 años, en que de $5.9 \%$ en 1950 ha alcanzado un $10 \%$ en 1967. Por otra parte, las defunciones por cáncer tienen mayor significación en el sexo femenino que en el masculino; para el mismo grupo de edades, 45-64 años, las proporciones relativas en 1967 , son 14.4 y $6.7 \%$, respectivamente.

\section{Mortalidad de origen cardiovascular}

Los datos que se refieren a este tipo de causas de defunción son aparentemente los más oscuros: el comportamiento experimentado entre 1950 y 1960 es distinto al de 1960-1967.30 En el primer período se muestra una tremenda reducción en la mortalidad por causas cardiovasculares en los primeros años (la tasa de mortalidad correspondiente pasa de 60 a 1 por cien mil en los menores de un año, y de 20 a 5 en las edades de 1 a 4 años); reducción que continúa hasta los 45 años, edad a partir de la cual se presentan ligeros incrementos de este género de mortalidad, lo que resulta en una estabilidad del nivel para todas las edades en conjunto. En el segundo periodo, en cambio, el nivel conjunto por causas cardiovasculares desciende: en 1967 es $60 \%$ menor que el de 1960 (o de 1950). Parece, pues, que existe algún cambio en los criterios de diagnóstico o de clasificación.

La experiencia histórica es que la mortalidad por estas causas disminuye, más en las mujeres que en los hombres, con el descenso de la mortalidad general. En México, los niveles de la mortalidad cardiovascular en 1950 se encontraban muy por debajo de lo esperado, por lo que es extraño el descenso que se presenta entre 1960 y 1967.

La resultante de 1950 a 1967 es un descenso de la mortalidad por causas cardiovasculares que abarca a todas las edades, con la excepción del grupo de 75 años y más, que no se altera. Este descenso, que entre los 45 y 74 años es más intenso en el sexo femenino, ocasiona un cambio que se convierte en una causa más de la sobremortalidad masculina (la mortalidad femenina, entre los 65 y 74 años, superaba a la masculina en 1950; en 1967 sucede lo contrario).

Un fenómeno que también es extraño a lo que se consideraría un

30 En el apéndice se encuentra información de años intermedios de estos dos períodos: 1955 y 1965. 
comportamiento esperado, se refiere a la proporción de las defunciones de origen cardiovascular; esta proporción, que dentro de las defunciones totales en las edades más avanzadas es significativa en otros países, en México es mucho menor: en las edades de 65 años y más estas defunciones sólo representan un $16 \%$ del total.

\section{Mortalidad por violencia y accidentes}

En el grupo IV de muertes violentas existe, por un lado, la influencia del adelanto general de la medicina, en cuanto que permite curas de accidentes mortales en épocas pasadas, y por otro, la aparición de nuevas formas de accidentes mortales surgidas del tipo de técnica alcanzada. Es motivo de controversia cuál sea la acción de un cierto desarrollo social sobre el balance final de homicidios y suicidios. En cambio, está fuera de duda el hecho universal de una mayor mortalidad masculina en este grupo de causas de muerte (explicable por el diferente comportamiento general de los sexos).

En México el fenómeno es bien patente, como ya se anotó anteriormente. A manera de ejemplo puede citarse que $\overline{e n}$ el año de 1967 el $47.8 \%$ de las defunciones masculinas tienen este origen, entre los 15 y 24 años, y el $35.7 \%$ entre los 25 y 44 años; comparado con 10.9 y $5.8 \%$ de las defunciones femeninas respectivas. Deberá notarse, además, que un porciento muy alto de este tipo de muertes se concentra en las edades centrales de la vida: en el caso de los hombres, $76.5 \%$ entre 15 y 64 años (tan sólo al grupo 25-44 años le correspondía un $39.2 \%$ ).

La mortalidad por violencia ha descendido alrededor de un $30 \%$ entre 1950 y 1967; siendo la disminución mayor entre los hombres $(34.5 \%)$ que entre las mujeres $(16 \%)$-tal vez debido a la gran diversidad en los niveles de mortalidad por violencia y accidentes-. Este descenso se presenta en la mayoría de los grupos centrales de edad. Sin embargo, por la mayor reducción de la mortalidad por otras causas (las del grupo I principalmente), este tipo de muertes continúa teniendo gran significación dentro del total de muertes, hasta las edades avanzadas -principalmente entre el sexo masculino.

\section{Mortalidad por las causas restantes}

El grupo V es un grupo residual y heterogéneo. Entre las defunciones en él incluidas "se encuentran causas de degeneración orgánica, como la diabetes, así como causas netamente exógenas como las complicaciones del parto".31 Este grupo incluye además el rubro B-45 (senilidad, causas mal definidas y desconocidas), de lo que resulta un elemento sumamente difícil de evaluar.

¿La mortalidad por "otras causas" ha descendido considerablemente en el lapso en cuestión $-35 \%$ en general-; el descenso es del orden de $69 \%$ en el grupo de 1-4 años, disminuyendo con la edad hasta un $15 \%$ en las edades de 75 años y mayores (en los menores de un año es de $41.5 \%$ ).

$$
\text { Signe ud uth }
$$

31 Naciones Unidas, op. cit., p. 107. 
La importancia relativa de estas "otras causas" dentro del total de causas de defunción asciende casi paralelamente al aumento del rubro B-45. Entre los menores de un año las causas restantes llegan a ser un $70 \%$ del total de defunciones; algo menos en los mayores de 65 años.

Cualquier comentario más amplio sería probablemente poco confiable. El porciento de muertes de las que se carece de información es un elemento que pone en entredicho todo lo comentado. Lo único plenamente seguro tal vez sólo sean los rasgos más generales de la evolución de la mortalidad por causas.

\section{CONSIDERACIONES FINALES}

La salud pública ha adelantado muy significativamente en México de 1930 a la fecha; sin embargo, parece ser que todavía un porciento muy elevado de las defunciones que ocurren en el país serían fácilmente evitables dado el tipo de muerte y las circunstancias en que ocurren. Los niveles y la proporción de defunciones por causas infecciosas y parasitarias, accidentes y violencia, causas insuficientemente especificadas y otras, son todavía en México muy cuantiosas en términos absolutos y en relación con niveles posibles de alcanzar si el desarrollo, en todos sus órdenes, se orientara no tan sólo cuantitativamente sino ante todo se le concibiera en términos cualitativos. ${ }^{32}$

Las anteriores observaciones sobre la estructura de la mortalidad por causas no son sino un marco de referencia, descriptivo, que deberá completarse con otros estudios de carácter más analítico o de enfoque más pragmático: diferencias regionales y sus causas en cuanto a la estructura de muertes; programas de salud pública; estudios de morbilidad; pérdidas de años-vida, económicas, etc.

32 Desde el punto de vista adoptado en el trabajo parece útil consignar algunas de las conclusiones de un estudio del Dr. Alfredo Heredia-Duarte donde afirma que "los adelantos que el pueblo mexicano ha experimentado en su salud, probablemente, no se deban más que en forma limitada a la asistencia médica", y, con base en sus hallazgos, "que la medicina institucional mexicana funciona deficientemente". Más adelante, después de consignar que las principales causas de muerte en México han sido, y continúan siendo las enfermedades infecciosas, afirma que "la desnutrición es una causa de enfermedad y muerte tan importante (como las principales causas de muerte en México), no obstante que su presencia no aparece consignada en las estadísticas de mortalidad." "Reflexiones sobre la medicina institucional mexicana", Gaceta Médica de Mé xico, Vol. 98, Núm. 4, abril de 1968, pp. 488-503. 


\section{APÉNDICE}

Cuadro A.1

MÉXICO: TASAS DE MORTALIDAd POR GRUPOS DE CAUSAS Y POR GRUPOS DE EDADES, 1950 (por 100000)

\begin{tabular}{|c|c|c|c|c|c|c|c|c|c|}
\hline \multirow{2}{*}{$\begin{array}{l}\text { Grupos de } \\
\text { cansas de } \\
\text { defunci } \delta n\end{array}$} & \multicolumn{9}{|c|}{ E d a d e $\mathrm{s}$} \\
\hline & Totales & 0 & $1-4$ & $5-14$ & $15-24$ & $25-44$ & $45-64$ & $65-74$ & $75 \mathrm{~g}$ más \\
\hline \multicolumn{10}{|c|}{ Ambos sexos } \\
\hline Totalea & 1615 & 13814 & 2769 & 350 & 433 & 781 & 1788 & 5102 & 13632 \\
\hline $\begin{array}{c}I \\
I I \\
I I I \\
I V \\
V\end{array}$ & $\begin{array}{r}580 \\
29 \\
94 \\
94 \\
818\end{array}$ & $\begin{array}{r}5722 \\
1 \\
60 \\
\\
726 \\
7905\end{array}$ & $\begin{array}{r}1264 \\
1 \\
20 \\
63 \\
1 \quad 421\end{array}$ & $\begin{array}{r}157 \\
1 \\
18 \\
29 \\
145\end{array}$ & $\begin{array}{r}150 \\
3 \\
30 \\
98 \\
152\end{array}$ & $\begin{array}{r}236 \\
21 \\
63 \\
150 \\
311\end{array}$ & $\begin{array}{l}504 \\
106 \\
238 \\
129 \\
811\end{array}$ & $\begin{array}{r}1250 \\
266 \\
785 \\
128 \\
2673\end{array}$ & $\begin{array}{r}2746 \\
378 \\
1688 \\
183 \\
8637\end{array}$ \\
\hline \multicolumn{10}{|c|}{ Hombres } \\
\hline Totales & 1717 & 14989 & 2734 & 357 & 492 & 892 & 2024 & 5310 & 13002 \\
\hline $\begin{array}{l}I \\
\text { II } \\
\text { III } \\
\text { IV } \\
V\end{array}$ & $\begin{array}{r}608 \\
21 \\
89 \\
159 \\
840\end{array}$ & $\begin{array}{r}6108 \\
1 \\
65 \\
4142 \\
8673\end{array}$ & $\begin{array}{r}1229 \\
2 \\
29 \\
69 \\
+413\end{array}$ & $\begin{array}{r}155 \\
1 \\
17 \\
39 \\
145\end{array}$ & $\begin{array}{r}151 \\
3 \\
26 \\
186 \\
126\end{array}$ & $\begin{array}{r}247 \\
11 \\
59 \\
283 \\
292\end{array}$ & $\begin{array}{r}584 \\
70 \\
250 \\
229 \\
891\end{array}$ & $\begin{array}{r}1386 \\
231 \\
758 \\
197 \\
2738\end{array}$ & $\begin{array}{r}2813 \\
332 \\
1471 \\
262 \\
8124\end{array}$ \\
\hline \multicolumn{10}{|c|}{ Mujeres } \\
\hline Totales & 1517 & 12648 & 2804 & 344 & 380 & 675 & 1561 & 4908 & 14173 \\
\hline $\begin{array}{c}I \\
\text { II } \\
\text { III } \\
\text { IV } \\
V\end{array}$ & $\begin{array}{r}553 \\
37 \\
99 \\
31 \\
797\end{array}$ & $\begin{array}{r}5342 \\
1 \\
\\
56 \\
110 \\
7 \quad 139\end{array}$ & $\begin{array}{r}1299 \\
1 \\
19 \\
19 \\
156 \\
1 \quad 429\end{array}$ & $\begin{array}{r}160 \\
1 \\
20 \\
19 \\
144\end{array}$ & $\begin{array}{r}150 \\
3 \\
33 \\
19 \\
175\end{array}$ & $\begin{array}{r}225 \\
31 \\
66 \\
24 \\
329\end{array}$ & $\begin{array}{r}426 \\
140 \\
227 \\
34 \\
734\end{array}$ & $\begin{array}{r}1124 \\
298 \\
811 \\
63 \\
2 \quad 612\end{array}$ & $\begin{array}{r}2689 \\
418 \\
1874 \\
115 \\
9077\end{array}$ \\
\hline
\end{tabular}

Fuente: Secretaría de Industria y Comercio, Dirección General de Estadística, "Defunciones por causas", copias mecanografiadas. 


\section{Cuadro A.2}

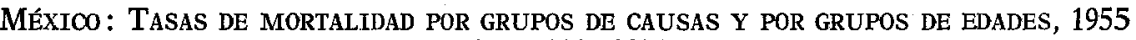
(por 100000 )

\begin{tabular}{|c|c|c|c|c|c|c|c|c|c|}
\hline \multirow{2}{*}{$\begin{array}{l}\text { Grupos de } \\
\text { causas de } \\
\text { defunción }\end{array}$} & \multicolumn{9}{|c|}{$E d a d e s$} \\
\hline & Totales & 0 & $1-4$ & $5-14$ & $15-24$ & $25-44$ & $45-64$ & $65-74$ & · 75 y más \\
\hline \multicolumn{10}{|c|}{ Ambos sexos } \\
\hline Totales & 1313 & 8957 & 2074 & 278 & 331 & 602 & 1458 & 4407 & 11122 \\
\hline $\begin{array}{c}I \\
I I \\
I I I \\
I V \\
V\end{array}$ & $\begin{array}{r}413 \\
28 \\
96 \\
82 \\
694\end{array}$ & $\begin{array}{r}3216 \\
1 \\
18 \\
83 \\
5 \quad 639\end{array}$ & $\begin{array}{r}905 \\
2 \\
18 \\
50 \\
1099\end{array}$ & $\begin{array}{r}113 \\
2 \\
14 \\
28 \\
121\end{array}$ & $\begin{array}{r}93 \\
3 \\
28 \\
87 \\
120\end{array}$ & $\begin{array}{r}150 \\
19 \\
60 \\
131 \\
242\end{array}$ & $\begin{array}{l}330 \\
104 \\
250 \\
116 \\
658\end{array}$ & $\begin{array}{r}841 \\
286 \\
905 \\
138 \\
2 \quad 237\end{array}$ & $\begin{array}{r}1760 \\
388 \\
1972 \\
186 \\
6816\end{array}$ \\
\hline \multicolumn{10}{|c|}{ Hombres } \\
\hline Totales & 1383 & 9561 & 2002 & 287 & 375 & $693^{\circ}$ & 1623 & 4569. & 10692 \\
\hline $\begin{array}{c}I \\
I I \\
I I I \\
I V \\
V\end{array}$ & $\begin{array}{r}428 \\
20 \\
.91 \\
133 \\
711\end{array}$ & $\begin{array}{r}3379 \\
1 \\
19 \\
19 \\
85 \\
6085\end{array}$ & $\begin{array}{r}866 \\
2 \\
18 \\
18 \\
52 \\
1064\end{array}$ & $\begin{array}{r}113 \\
2 \\
13 \\
38 \\
121\end{array}$ & $\begin{array}{r}89 \\
3 \\
25 \\
159 \\
99\end{array}$ & $\begin{array}{r}159 \\
17 \\
56 \\
242 \\
225\end{array}$ & $\begin{array}{r}378 \\
69 \\
260 \\
198 \\
718\end{array}$ & $\begin{array}{r}919 \\
238 \\
888 \\
216 \\
2308\end{array}$ & $\begin{array}{r}1782 \\
341 \\
1792 \\
258 \\
6519\end{array}$ \\
\hline \multicolumn{10}{|c|}{ Mujeres } \\
\hline Totales & 1243 & 8320 & 2150 & 269 & 291 & 515 & 1294 & 4252 & 11502 \\
\hline $\begin{array}{c}I \\
I I \\
I I I \\
I V \\
V\end{array}$ & $\begin{array}{r}398 \\
35 \\
102 \\
31 . \\
677\end{array}$ & $\begin{array}{rr}3052 \\
& 1 \\
& 17 \\
& 82 \\
5 & 168\end{array}$ & $\begin{array}{r}946 \\
2 \\
20 \\
48 \\
1434\end{array}$ & $\begin{array}{r}113 \\
2 \\
15 \\
19 \\
120\end{array}$ & $\begin{array}{r}98 \\
3 \\
31 \\
20 \\
139\end{array}$ & $\begin{array}{r}142 \\
28 \\
64 \\
24 \\
257\end{array}$ & $\begin{array}{r}282 \\
137 \\
240 \\
36 \\
599\end{array}$ & $\begin{array}{r}767 \\
332 \\
921 \\
64 \\
2.168\end{array}$ & $\begin{array}{r}1740 \\
429 \\
2139 \\
124 \\
7078\end{array}$ \\
\hline
\end{tabular}

Fuente: Secretaría de Industria y Comercio, Dirección General de Estadística, "Defunciones según causas", copias mecanografiadas. 
Cuadro A.3

MÉXICO: TASAS DE MORTALIDAD POR GRUPOS DE CAUSAS Y POR GRUPOS DE EDADES, 1960 (por 100000 )

\begin{tabular}{|c|c|c|c|c|c|c|c|c|}
\hline \multirow{2}{*}{$\begin{array}{l}\text { Grupos de } \\
\text { causas de } \\
\text { defunci } 8 n\end{array}$} & \multicolumn{8}{|c|}{$E d a d \theta$} \\
\hline & Totales & 0 & $1-4$ & 514 & $15-24$ & $25-44$ & $45-64$ & $65 \mathrm{y}$ máx \\
\hline \multicolumn{9}{|c|}{ Ambos sexos } \\
\hline Totales & $1+49$ & 10217 & 1447 & 209 & 273 & 543 & 1417 & 5650 \\
\hline $\begin{array}{c}I \\
I I \\
I I I \\
I V \\
V\end{array}$ & $\begin{array}{r}314 \\
36 \\
97 \\
75 \\
627\end{array}$ & $\begin{array}{r}3 \quad 241 \\
3 \\
\\
4 \\
60 \\
6892\end{array}$ & $\begin{array}{r}599 \\
2 \\
5 \\
43 \\
798\end{array}$ & $\begin{array}{r}74 \\
2 \\
70 \\
25 \\
98\end{array}$ & $\begin{array}{r}64 \\
5 \\
22 \\
77 \\
105\end{array}$ & $\begin{array}{r}117 \\
23 \\
57 \\
120 \\
226\end{array}$ & $\begin{array}{l}278 \\
131 \\
269 \\
115 \\
624\end{array}$ & $\begin{array}{r}1073 \\
412 \\
1381 \\
152 \\
3632\end{array}$ \\
\hline \multicolumn{9}{|c|}{ Hombreg } \\
\hline Totales & 1216 & 11150 & 1409 & 217 & 307 & 626 & 1587 & 6542 \\
\hline $\begin{array}{c}I \\
\text { II } \\
\text { III } \\
\text { IV } \\
V\end{array}$ & $\begin{array}{r}328 \\
28 \\
92 \\
121 \\
647\end{array}$ & $\begin{array}{r}3468 \\
3 \\
2 \\
2 \\
87 \\
7 \quad 590\end{array}$ & $\begin{array}{r}577 \\
3 \\
5 \\
46 \\
778\end{array}$ & $\begin{array}{r}74 \\
3 \\
9 \\
34 \\
97\end{array}$ & $\begin{array}{r}59 \\
5 \\
19 \\
137 \\
87\end{array}$ & $\begin{array}{r}125 \\
13 \\
53 \\
221 \\
214\end{array}$ & $\begin{array}{r}325 \\
94 \\
282 \\
192 \\
694\end{array}$ & $\begin{array}{l}1110 \\
380 \\
1283 \\
213 \\
3556\end{array}$ \\
\hline \multicolumn{9}{|c|}{ Mujeren } \\
\hline Totales & 1083 & 9559 & 1477 & 202 & 241 & 463 & 1249 & 6752 \\
\hline $\begin{array}{c}I \\
I I \\
I I I \\
I V \\
V\end{array}$ & $\begin{array}{r}300 \\
44 \\
103 \\
29 \\
607\end{array}$ & $\begin{array}{rr}3105 \\
3 \\
\\
\\
\\
6 \quad 375\end{array}$ & $\begin{array}{r}618 \\
2 \\
6 \\
39 \\
812\end{array}$ & $\begin{array}{r}73 \\
2 \\
10 \\
17 \\
100\end{array}$ & $\begin{array}{r}68 \\
5 \\
25 \\
20 \\
123\end{array}$ & $\begin{array}{r}109 \\
32 \\
61 \\
23 \\
238\end{array}$ & $\begin{array}{r}231 \\
168 \\
258 \\
38 \\
554\end{array}$ & $\begin{array}{r}1039 \\
442 \\
1472 \\
95 \\
3704\end{array}$ \\
\hline
\end{tabular}

Fuente: Secretaría de Salubridad y Asistencia, Anuario Estadístico de Salubridad y Asistencia. 


\section{Cuadro A.4}

México: TASAS DE MORTALIDAd POR GRUPOS DE CAUSAS Y POR GRUPOS DE EDADES, 1965 (por 100000)

\begin{tabular}{|c|c|c|c|c|c|c|c|c|c|}
\hline \multirow{2}{*}{$\begin{array}{l}\text { Grupos de } \\
\text { causas de } \\
\text { defunción } \\
\end{array}$} & \multicolumn{9}{|c|}{$\mathrm{E} d \approx \mathrm{d}$ e $\mathrm{g}$} \\
\hline & Totales & 0 & $1-4$ & $5-14$ & $15-24$ & $25-44$ & $45-64$ & $65-74$ & $75 \mathrm{y}$ más \\
\hline \multicolumn{10}{|c|}{ Ambos sexos } \\
\hline Totales & 944 & 6542 & 941 & 170 & 223 & 470 & 1293 & 3716 & 11804 \\
\hline $\begin{array}{c}I \\
I I \\
I I I \\
I V \\
V\end{array}$ & $\begin{array}{r}228 \\
36 \\
64 \\
66 \\
550\end{array}$ & $\begin{array}{r}1989 \\
3 \\
8 \\
\\
454 \\
4 \quad 488\end{array}$ & $\begin{array}{r}393 \\
3 \\
4 \\
36 \\
505\end{array}$ & $\begin{array}{r}53 \\
3 \\
6 \\
24 \\
84\end{array}$ & $\begin{array}{r}39 \\
5 \\
12 \\
68 \\
99\end{array}$ & $\begin{array}{r}80 \\
23 \\
33 \\
106 \\
228\end{array}$ & $\begin{array}{l}197 \\
133 \\
180 \\
105 \\
678\end{array}$ & $\begin{array}{r}537 \\
352 \\
610 \\
132 \\
2085\end{array}$ & $\begin{array}{r}1579 \\
632 \\
1760 \\
214 \\
7619\end{array}$ \\
\hline \multicolumn{10}{|c|}{ Hombres } \\
\hline Totales & 998 & 7093 & 917 & 177 & 247 & 551 & 1500 & 3972 & $1102 ุ 1$ \\
\hline $\begin{array}{c}I \\
I I \\
I I I \\
I V \\
V\end{array}$ & $\begin{array}{r}239 \\
29 \\
61 \\
104 \\
565\end{array}$ & $\begin{array}{rr}2116 \\
. \quad 3 \\
\\
9 \\
60 \\
4 \quad 905\end{array}$ & $\begin{array}{r}375 \\
4 \\
4 \\
39 \\
495\end{array}$ & $\begin{array}{r}53 \\
3 \\
6 \\
32 \\
83\end{array}$ & $\begin{array}{r}38 \\
5 \\
10 \\
115 \\
79\end{array}$ & $\begin{array}{r}88 \\
14 \\
32 \\
194 \\
223\end{array}$ & $\begin{array}{l}244 \\
102 \\
197 \\
178 \\
779\end{array}$ & $\begin{array}{r}615 \\
330 \\
640 \\
192 \\
2195\end{array}$ & $\begin{array}{r}1551 \\
1594 \\
1529 \\
270 \\
7077\end{array}$ \\
\hline \multicolumn{10}{|c|}{ Mujereg } \\
\hline Totales & 889 & 5967 & 966 & 164 & 198 & 393 & 1097 & 3476 & 12528 \\
\hline $\begin{array}{c}I \\
I I \\
I I I \\
I V \\
V\end{array}$ & $\begin{array}{r}217 \\
43 \\
66 \\
27 \\
536\end{array}$ & $\begin{array}{r}1855 \\
4 \\
4 \\
4 \\
4 \\
4 \quad 054\end{array}$ & $\begin{array}{r}411 \\
3 \\
3 \\
33 \\
516\end{array}$ & $\begin{array}{r}53 \\
3 \\
7 \\
16 \\
85\end{array}$ & $\begin{array}{r}41 \\
5 \\
13 \\
19 \\
120\end{array}$ & $\begin{array}{r}73 \\
31 \\
33 \\
23 \\
233\end{array}$ & $\begin{array}{r}153 \\
162 \\
163 \\
36 \\
583\end{array}$ & $\begin{array}{r}463 \\
374 \\
581 \\
75 \\
1983\end{array}$ & $\begin{array}{r}1605 \\
668 \\
1 \quad 773 \\
163 \\
8119\end{array}$ \\
\hline
\end{tabular}

Fuente: Secretaría de Salubridad y Asistencia, Dirección de Bioestadística, Estadisticas Vitales de los Estados Unidos Mexicanos. 
Cuadro A.5

MÉXICO: TASAS DE MORTalidad POR GRUPOS DE CAUSAS Y POR GRUPOS DE EDADES, 1967 (por 100000 )

\begin{tabular}{|c|c|c|c|c|c|c|c|c|c|}
\hline \multirow{2}{*}{$\begin{array}{l}\text { Grupos de } \\
\text { causas de } \\
\text { defuncibn } \\
\end{array}$} & \multicolumn{9}{|c|}{$E d a d e B$} \\
\hline & Totales & 0 & $1-4$ & $5-14$ & $15-24$ & $25-44$ & $45-64$ & $65-74$ & 75 y más \\
\hline \multicolumn{10}{|c|}{ Ambos sexos } \\
\hline Totales & 911 & 6769 & 854 & 156 & 211 & 457 & +236 & 3689 & 11220 \\
\hline $\begin{array}{c}I \\
I I \\
I I I \\
I V \\
V\end{array}$ & $\begin{array}{r}218 \\
34 \\
63 \\
65 \\
531\end{array}$ & $\begin{array}{r}2088 \\
\\
\\
\\
\\
\\
4 \quad 53 \\
4 \quad 619\end{array}$ & $\begin{array}{r}372 \\
3 \\
3 \\
34 \\
442\end{array}$ & $\begin{array}{r}47 \\
3 \\
6 \\
24 \\
76\end{array}$ & $\begin{array}{r}35 \\
5 \\
10 \\
67 \\
94\end{array}$ & $\begin{array}{r}73 \\
21 \\
31 \\
106 \\
226\end{array}$ & $\begin{array}{l}173 \\
124 \\
176 \\
105 \\
658\end{array}$ & $\begin{array}{r}481 \\
350 \\
659 \\
134 \\
2065\end{array}$ & $\begin{array}{r}411 \\
603 \\
1705 \\
206 \\
7295\end{array}$ \\
\hline \multicolumn{10}{|c|}{ hombres } \\
\hline Totales & 969 & 7298 & 838 & 164 & 237 & 547 & 1452 & 4034 & 10427 \\
\hline $\begin{array}{l}I \\
\text { II } \\
\text { III } \\
\text { IV } \\
V\end{array}$ & $\begin{array}{r}228 \\
28 \\
61 \\
104 \\
548\end{array}$ & $\begin{array}{r}2203 \\
2 \\
\\
\\
\\
56 \\
5031\end{array}$ & $\begin{array}{r}357 \\
4 \\
3 \\
39 \\
435\end{array}$ & $\begin{array}{r}46 \\
3 \\
6 \\
32 \\
77\end{array}$ & $\begin{array}{r}33 \\
5 \\
9 \\
113 \\
77\end{array}$ & $\begin{array}{r}81 \\
13 \\
30 \\
196 \\
227\end{array}$ & $\begin{array}{r}213 \\
98 \\
200 \\
182 \\
7.59\end{array}$ & $\begin{array}{r}559 \\
343 \\
719 \\
207 \\
2206\end{array}$ & $\begin{array}{r}1387 \\
1560 \\
1489 \\
256 \\
6735\end{array}$ \\
\hline \multicolumn{10}{|c|}{ Mujeres } \\
\hline Totales & 853 & 6216 & 872 & 147 & 1.85 & 373 & 1034 & 3367 & 11955 \\
\hline $\begin{array}{c}I \\
\text { II } \\
\text { III } \\
\text { IV } \\
V\end{array}$ & $\begin{array}{r}208 \\
40 \\
64 \\
26 \\
515\end{array}$ & $\begin{array}{r}1967 \\
\\
\\
\\
\\
\\
45 \\
4 \quad 51 \\
4\end{array}$ & $\begin{array}{r}387 \\
3 \\
3 \\
30 \\
449\end{array}$ & $\begin{array}{r}48 \\
3 \\
6 \\
15 \\
75\end{array}$ & $\begin{array}{r}37 \\
5 \\
12 \\
20 \\
111\end{array}$ & $\begin{array}{r}66 \\
29 \\
32 \\
22 \\
224\end{array}$ & $\begin{array}{r}135 \\
149 \\
153 \\
33 \\
564\end{array}$ & $\begin{array}{r}409 \\
356 \\
603 \\
66 \\
1933\end{array}$ & $\begin{array}{r}1433 \\
642 \\
1906 \\
160 \\
7814\end{array}$ \\
\hline
\end{tabular}

Fuente: Secretaría de Salubridad y Asistencia, Dirección de Bioestadística, Estadísticas Vitales de los Estados Unidos Mexicanos. 
Cuadro A.6

México: Cambios EN La MORTALIdAd POR GRUPOS DE CAUSAS, 1950-1967

(cambios porcentuales)

\begin{tabular}{|c|c|c|c|c|c|c|c|c|c|}
\hline \multirow{2}{*}{$\begin{array}{l}\text { Grupos de } \\
\text { causas de } \\
\text { defunción } \\
\end{array}$} & \multicolumn{9}{|c|}{ E $d a d e \mathbf{s}$} \\
\hline & Totales & 0 & $1-4$ & $5-14$ & $15-24$ & $25-44$ & $45-64$ & $65-74$ & $75 y+$ \\
\hline \multicolumn{10}{|c|}{ Ambos sexos } \\
\hline Totales & -43.59 & -51.00 & -69.16 & -55.43 & -51.27 & -41.49 & -30.87 & -27.70 & -17.69 \\
\hline $\begin{array}{c}I \\
I I \\
I I I \\
I V \\
V\end{array}$ & $\begin{array}{r}-62.41 \\
17.24 \\
-32.98 \\
-30.85 \\
-35.09\end{array}$ & $\begin{array}{l}-63.51 \\
200.00 \\
-90.00 \\
-57.94 \\
-41.57\end{array}$ & $\begin{array}{l}-70.57 \\
200.00 \\
-85.00 \\
-46.03 \\
-68.90\end{array}$ & $\begin{array}{l}-70.06 \\
200.00 \\
-66.67 \\
-17.24 \\
-47.59\end{array}$ & $\begin{array}{r}-76.67 \\
66.67 \\
-66.67 \\
-31.63 \\
-38.16\end{array}$ & $\begin{array}{r}-69.07 \\
0.00 \\
-50.79 \\
-29.33 \\
-27.33\end{array}$ & $\begin{array}{r}-65.67 \\
16.98 \\
-26.05 \\
-18.60 \\
-18.87\end{array}$ & $\begin{array}{r}-61.52 \\
31.58 \\
-16.05 \\
4.69 \\
-22.75\end{array}$ & $\begin{array}{r}-48.62 \\
59.52 \\
1.01 \\
12.57 \\
-15.54\end{array}$ \\
\hline \multicolumn{10}{|c|}{ Hombres } \\
\hline Totales & -43.56 & -51.31 & -69.35 & -54.06 & -51.83 & -38.68 & -28.26 & -24.03 & -19.80 \\
\hline $\begin{array}{c}I \\
I I \\
I I I \\
I V \\
V\end{array}$ & $\begin{array}{r}-62.50 \\
33.33 \\
-31.46 \\
-34.59 \\
-34.76\end{array}$ & $\begin{array}{l}-63.93 \\
100.00 \\
-90.77 \\
-60.56 \\
-41.99\end{array}$ & $\begin{array}{l}-70.95 \\
100.00 \\
-85.71 \\
-43.48 \\
-69.21\end{array}$ & $\begin{array}{l}-70.32 \\
200.00 \\
-64 \cdot 71 \\
-17.95 \\
-46.90\end{array}$ & $\begin{array}{r}-78.15 \\
66.67 \\
-65.38 \\
-39.25 \\
-38.89\end{array}$ & $\begin{array}{r}-67.21 \\
18.18 \\
-49.15 \\
-30.74 \\
-22.26\end{array}$ & $\begin{array}{r}-63.53 \\
40.00 \\
-20.00 \\
-20.52 \\
-14.81\end{array}$ & $\begin{array}{r}-59.67 \\
48.48 \\
-5.15 \\
5.08 \\
-19.43\end{array}$ & $\begin{array}{r}-50.69 \\
68.67 \\
1.22 \\
-2.29 \\
-17.10\end{array}$ \\
\hline \multicolumn{10}{|c|}{ Mujeres } \\
\hline Totales & -43.77 & -50.85 & -68.90 & $-57 \cdot 27$ & -51.32 & $-44 \cdot 74$ & $-33 \cdot 76$ & -31.40 & -15.65 \\
\hline $\begin{array}{c}I \\
I I \\
I I I \\
I V \\
V\end{array}$ & $\begin{array}{r}-62.39 \\
8.11 \\
-35.35 \\
-16.13 \\
-35.38\end{array}$ & $\begin{array}{l}-63.18 \\
200.00 \\
-91.07 \\
-53.64 \\
-41.31\end{array}$ & $\begin{array}{l}-70.21 \\
200.00 \\
-84.21 \\
-46.43 \\
-68.58\end{array}$ & $\begin{array}{l}-70.00 \\
200.00 \\
-70.00 \\
-21.05 \\
-47.92\end{array}$ & $\begin{array}{r}-75.33 \\
66.67 \\
-63.64 \\
5.26 \\
-36.57\end{array}$ & $\begin{array}{l}-70.67 \\
-6.45 \\
-51.52 \\
-8.33 \\
-31.91\end{array}$ & $\begin{array}{r}-68.31 \\
6.43 \\
-32.60 \\
-2.94 \\
-23.16\end{array}$ & $\begin{array}{r}-63.61 \\
19.46 \\
-25.65 \\
4.76 \\
-26.00\end{array}$ & $\begin{array}{r}-46.71 \\
53.59 \\
1.71 \\
39.13 \\
-13.91\end{array}$ \\
\hline
\end{tabular}

Cuadro A.7

MÉXico: Distribución DE LAS DEFUnCIONES POR SEXo Y GRUPOS DE EDAD, $1950,1960,1967$

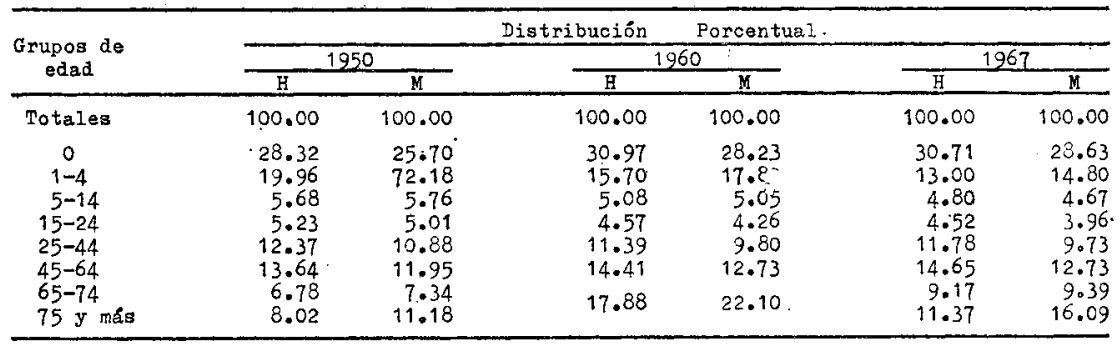

